\title{
VOLUME XIII
}

\section{INDEX OF SUBJECTS AND AUTHORS}

Only main topics are listed, and reference is given to the

first page of each paper in which they are discussed

Abortive recombinants in $\mathrm{I}^{+} \times \mathrm{I}^{+}$bacterial crosses, 113

Acridines, genetic effect of, in Pseudomonas aeruginosa, 57

Acriflavine resistant rII deletions in bacteriophage T 4, 329

Ascus development mutants in Neurospora crassa, 303

Attached-X deletion, lethal effects of in Drosophila melanogaster, 325

Bacteriophage T4, acriflavine resistant rII deletions, 329

Baker, L. H., 105

Basl, Mary, 303

Beisson, Janine, 85

Bell, A. E., 175

Bevan, E. A., 71

Braver, Gerald, 325

Burnet, Barrie, 251

Byrne, Barbara J., 197

Chromosomal polymorphism in Spalax leucodon, 99

Crow, James, F., 127

Chrysanthemum, nuclei acid content and chromosomal morphology of, 241

Colson, C., 227

Cost of natural selection, a vindication of Haldane's original theory despite complicating factors, 127

Curnow, R. N., 105

Curtis, C. F., 289

Cytoplasmic factor in killer phenotype in Saccharomyces cerevisiae, 71

Cytoplasmic respiratory deficiency induced by phenethyl elcohol in Saccharomyces cerevisiae, 107

Cytogenetic study of Spalax leucodon in Moldavia, 99

Dickinson, A. G., 213

Dowrick, G. J., 241

Drosophila melanogaster gene-environment interactions of eye gene, 313 gene-environment interactions of eyeless, 251

lethal effects of attached-X deletion, 325

nutritional conditional mutants, 281

Duma, Doina, 99

Edwards, Susan, 321

El Bayoumi, A. S., 241

Episomal state, demonstration of, with genes in Staphylococcus aureus, 267

Escherichia coli

genetics of host-controlled restriction and modification, 227

$\mathrm{I}^{+} \times \mathrm{I}^{+}$crosses and unstable products, 113

Falconer, D. S., 25

Fertility, and ovulation rate in mouse, 25

Gene-environment interactions in eyeless and eye gene mutants of Drosophila melano. gaster, 251,313

Genetic disturbance after species transfer of linkage group $\mathrm{H}_{2} \mathrm{Lc}_{2}$ in Gossypium, 1

Glossina austeni, production of partially sterile mutants, 289

Glover, S. W., 227

Gossypium, genetic disturbance of linkage group after species transfer, 1

Hill, W. G., 143, 165

Holloway, B. W., 57

Host-controlled modification and restriction in Escherichia coli, 227

Hunt, David, M., 251, 313

I sex factors and chromosomal recombination in Salmonella typhimurium, 321

Johnson, D. R., 275

Johnston, Joan, 267

Kappa, mu and the metagon in Paramecium aurelia, 197

Killer character in Saccharomyces cerevisiae, 71 
Kimura, Motoo, 47, 127

Krzanowska, H., 17

Land, R. B., 25

Linkage

disequilibrium due to random genetic drift, 47

$\mathrm{H}_{2} \mathrm{Lc} c_{2}$ in Gossypium, reduction of recombination after species transfer, 1

of thermosensitive mutants in Paramecium aurelia, 85

Loutit, J. S., 91

Mapping, of distal markers in Pseudomonas aeruginosa, 91

of sites for host-controlled modification and restriction, 227

Maroudas, N. G., 107

Meikle, Veronica M. H., 213

Metagon hypothesis in Paramecium aurelia, lack of compatibility with results, 197

Meynell, G. G., 113, 327

Mouse

brachyphalangy, an allele of extra-toes, 275

comparison of biological characters of scrapie agents $22 \mathrm{~A}$ and $\mathrm{ME} \mathrm{7,213}$

factor for spermatozoan abnormality on $Y$-chromosome, 17

ovulation rate in, 25

Mutant

brachyphalangy, an allele of extra-toes in mouse, 275

eyeless and eye gene and environmental interactions, in Drosophila melanogaster, 251,313

production of partially sterile mutants of Glossina austeni, 289

Nash, David, 281

Natural selection, cost of, 127

Neurospora crassa mutants affecting ascus development, 303

Nucleic acid content and chromosome morphology in Chrysanthemum, 241

Nutritional conditional mutants of Drosophila melanogaster, 281

Ohta, Tomoko, 47

Ovulation rate in mouse, 25

Paramecium aurelia, a linkage group in, 85

kappa, mu and the metagon, 197

Parma, David H., 329

Pike, Derek, J., 117
Plasmids as episomes in Staphylococcus aureus, 267

Polyploids, DNA content of natural and induced, in Chrysanthemum, 241

Probability transition matrices, and selection of finite populations, 143

versus continuous distribution as methods of predicting gene frequency shifts in selected finite populations, 117

Pseudomonas aeruginosa genetic effect of acridines, 57

mapping of distal markers, 91

Raicu, Petre, 99

Random genetic drift, 47

Recombination, reduction of values on species transfer, in Gossypium, 1

Repeated selection in finite populations: a comparison of methods of detection, 117

Richmond, M. H., 267

Rossignol, Michele, 85

Saccharomyces cerevisiae

induction of cytoplasmic respiratory deficiency with phenethyl alcohol, 107

inheritance of killer character, 71

Salmonella typhimurium

$\mathrm{I}^{+} \times \mathrm{I}^{+}$crosses and unstable products, 113

I sex factor and chromosomal recombination, 321

Saunders, J. H., 1

Scrapie, comparison of biological properties of $22 \mathrm{~A}$ and $\mathrm{ME} 7$ in mouse, 213

Selection

artificial in finite populations, 143

effect of repeated cycles in populations of finite size, 105

for larval growth under two levels of nutrition in Tribolium castaneum, 175

for natural and induced ovulation rates in mouse, 25

natural, and gene substitution, 127

rate of advance for non-additive loci, 165

Sex factor of Pseudomonas aeruginosa and effect of acridines, 57

Somers, J. M., 71

Spalax leucodon, cytogenetic studies of, 99

Spermatozoan abnormality, a factor on $Y$-chromosomes in mouse, 17

Srb, Adrian M., 303

Stanisich, Vilma, 57

Staphylococcus aureus, reversible transition between episomal states, 267

Superinfection immunity in $\mathrm{I}^{+} \times \mathrm{I}^{+}$crosses in bacteria, 113 
Thermosensitive mutants in a linkage group Wilkie, D., 107 in Paramecium aurelia, 85

Thomson, James N. Jun., 325

Yamada, Yukio, 175

Tribolium castaneum, selection for larval growth on two levels of nutrition, 175

$Y$-chromosome factor for sperm abnormality in mouse, 17

Vyse, E. R., 281 